\title{
Karyotype Learning Center: A Software For Teaching And Learning Cytogenetics
}

\author{
De Mesquita, Joelma Freire ${ }^{1}$; dos Santos, Suely $R^{2}{ }^{2}$ Paiva, Carmen Lucia A. ${ }^{2}$ \\ ${ }^{1}$ Departamento de Bioquímica - IQ/USP; ${ }^{2}$ Unidade de Genética - UNIRIO
}

The in vitro cultivation of human cells is an essential part of the work of every diagnostic cytogenetics laboratory. Almost all human cytogenetic studies involve the examination of dividing blood cell population by blocking cell division at metaphase with subsequent processing and staining by banding techniques. The chromosome constitution is described as Karyotype that states the total number of chromosomes and the sex chromosome constitution. Karyotypes are prepared by cutting up a photograph of the spread metaphase chromosomes, matching up homologous chromosomes and sticking them back down on a card or nowadays more often by getting an image analysis computer to do the job. Chromosomes are identified by their size, centromere position and banding pattern. Teaching a student how to detect and interpret even the most common chromosome abnormalities is a major challenge: mainly, in a developing country where the laboratorial facilities are not always available for a big number of students. Therefore, in this work we present an educational software for teaching undergraduate students of Medical and Life Sciences Courses how to arrange normal and abnormal chromosomes in the form of karyotype. The user, using drag-and-drop, is dared to match up homologous chromosome. For that, we have developed a free full access web site (http://www.biomol.net/cariotipo/) for hosting the software. The latter has proved to be light and fast even under slow dial-up connections. This web site also offers a theoretical introductory section with basic concepts about karyotype. Up to now the software has been successfully applied to undergraduate courses at the University of Rio de Janeiro (UNIRIO). The students have approved the software; to them the similarities with the well-known game solitaire turns the exercise more exciting and provides additional stimulus to learn and understand karyotype. Professors have also used the software as complementary material in their regular classes. Based on their testimonies the software was a helpful tool for explaining the karyotype assembly. Financial support: FAPESP, UNIRIO. 